\title{
The Effect of Regular Aerobic Exercise on Reverse Cholesterol Transport A1 and Apo Lipoprotein A-I Gene Expression in Inactive Women
}

\author{
Asghar Tofighi ${ }^{1}$; Fatemeh Rahmani ${ }^{2}$; Bahram Jamali Qarakhanlou ${ }^{3}$; Solmaz Babaei ${ }^{1, *}$ \\ ${ }_{1}^{1}$ Department of Exercise Physiology, Faculty of Physical Education and Sport Sciences, Urmia University, Urmia, IR Iran \\ ${ }^{2}$ Institute of Biotechnology, Urmia University, Urmia, IR Iran \\ 3 Department of Exercise and Sports Sciences, Tabriz University of Medical Sciences, Tabriz, IR Iran \\ ${ }^{*}$ Corresponding Author: Solmaz Babaei, Department of Exercise Physiology, Faculty of Physical Education and Sport Sciences, Urmia University, Urmia, IR Iran. Tel: +98-9143215066, \\ Fax:+98-4133369696, E-mail: so_babaei@yahoo.com
}

Received: December 22, 2014; Accepted: January 28, 2015

\begin{abstract}
Background: Atherosclerotic cardiovascular disease is currently a cause of mortality in some parts of the world. The ATP-Binding Cassette Transporter (ABCA1) gene prepares instructions to produce the ATP-binding cassette transporter protein whose operation is for export of phospholipids and cholesterol, outside cells where they are limited to Apolipoprotein A1 (apoA1). Increased ABCA1 activity could inhibit atherosclerosis.

Objectives: In the present study, the effect of aerobic exercise was investigated on gene expression and biochemical parameters.

Patients and Methods: The participants included 36 inactive women, which were randomly assigned to control(CON)and experimental (EX) groups. The EX group performed 12 weeks of aerobic exercise and the CON group remained inactive. Fasting blood samples were collected 24 hours before the first session and 48 hours after completion of the course. The ABCA1 and APOA1 gene expressions were measured using semi-quantitative-RT-PCR. Data were analyzed by the SPSS software (version 18).

Results: A significant increase in blood ABCA1 (EX group $\mathrm{P}<0.002, \mathrm{t}=-9.876$ ) and Apo A-I (EX group $\mathrm{P}<0.05, \mathrm{t}=2.76$ ) gene expression was shown following the 12 weeks of training. Plasma high-density lipoprotein-cholesterol (HDL-C) concentration increased $(\mathrm{P}<0.001, \mathrm{t}=$ 4.90 respectively) while plasma low-density lipoprotein-cholesterol (LDL-C) concentration decreased $(\mathrm{P}<0.001, \mathrm{t}=4.27)$ in the EX group compared with the CON group.

Conclusions: Aerobic exercises can increase ABCA1 and APO-A1 gene expression. Induction of these genes can effectively prevent cardiovascular disease.
\end{abstract}

Keywords: APOA1; Aerobic Exercises; Cholesterol

\section{Background}

Coronary artery disease is currently a major cause of mortality worldwide. The primary symptom of atherosclerosis is the accumulation of foam cells in the intima of arteries. An elevated plasma level of Low-Density Lipoprotein (LDL) is a risk factor for atherosclerosis, mainly because this lipoproteins deposit cholesterol within cells of the arterial wall (1). Also, multiple epidemiological studies have shown that plasma level of High-Density Lipoproteins (HDL), and their main protein constitutive Apolipoprotein A-I (apoA-I) are inversely correlated with the risk of atherosclerosis. In addition, the rise of $\mathrm{HDL}$ cholesterol prevents atherogenesis in multiple genetic animal models (1). This protective effect of HDL-C against atherosclerosis (2) is due to the role of HDL-C in Reverse Cholesterol Transport (RCT). In RCT, HDL-C intermediates the elimination of excess free cholesterol from peripheral cells to liver for secretion as bile (3). In RCT, the production and reconstruction of HDL-C in plasma requires various factors, including ATP-binding cassette transporters, especially ABCA1, known as one of the key elements of the reverse cholesterol transport pathway $(4,5)$. ABCA1 is abundantly expressed in the liver, brain macrophages and other different tissues $(6,7)$ and facilitates delivery of phospholipids from cell membranes to lipid-poor Apo A-1 with the formation of Apo A-I with HDL. Hence, it plays a key role in plasma HDL formation $(8,9)$. ABCA1 is necessary for the preservation of plasma HDL-C levels. Generally, it is accepted that an increase in liver ABCA1 expression will have a strong effect on formation of plasma HDL-C to protect against atherosclerosis $(10,11)$. ABCA1 is responsible for lipidation of lipid-poor Apo A-I by cellular cholesterol and phospholipids, a rate-limiting process in both formation of HDL and cholesterol efflux (12). Peripheral factors significantly contribute to changes in ABCA1 expression and plasma HDL-C in the general population (13).

Previous reports show that an increase of Apo-A release in the liver and small intestine and greater ABCA1 expression in macrophages have a strong effect on RCT process, formation of plasma HDL-C and protection against ath- 
erosclerosis (5). Moreover, ABCA1 might play a role in inflammation and is unregulated by several factors such as physical activity, cholesterol influx, plasma glucose concentration and nutritional status (14). So far, only three studies have focused on the effect of physical activity on $\mathrm{ABCA1}$ expression $(12,15,16)$. In the first study, ABCA1expression was measured in human skeletal muscle biopsies and blood with assessment of physical activity (habitual exercise) using the International Physical Activity Questionnaire (IPAQ) (12). In the second research, conducted by Butcher et al. the effect of low-intensity exercise training (eight weeks of low-intensity exercise program consisting of walking 10000 steps, three times a week) was studied on blood ABCA1and ABCG1 expression (16). The third study showed that a single circuit resistance exercise (nine exercises, 25 seconds per exercise, three sets of three without interruption circuits and one minute rest interval between the sets) at three given intensities (40\%, 60\% and $80 \%$ one-repetition maximum) increased ABCA1 expression in peripheral blood (15). To the best of our knowledge, no study has focused on the effect of aerobic exercise on blood ABCA1and APOA1 in obese women.

\section{Objectives}

Thus, this study was conducted to investigate blood ABCA1 expression in response to 12 weeks of aerobic exercise in inactive women.

\section{Patients and Methods}

\subsection{Study Subjects}

All aspects of the use of human subjects in this study were approved by the Ethical Committee of Urmia University, Urmia, Iran. Written informed consent was obtained from all subjects in accordance with the principles of the Helsinki Declaration. Thirty-six inactive woman were randomly assigned to CON $(n=18)$ and EX $(n=18)$ groups. Participants were asked to complete medical tests and the health questionnaire to ascertain that they did not use any kind of drug, and were free of cardiorespiratory, renal and metabolic diseases. The EX group performed three sessions (morning)/week of aerobic exercise for 12 weeks and the CON group remained sedentary during this period. All participants were accustomed to the training protocol.

\subsection{Training Protocol}

The exercise program lasted for 12 weeks, three sessions a week with intensity of between 65 to $75 \%$ of participants' maximum heart rate. Participants in the first three weeks, the fourth and fifth, and ultimately from sixth to twelfth week exercised with $0.65,0.70$ and 0.75 maximum heart rate, respectively. The $0.65,0.70$ and 0.75 maximum heart rates were related to the original exercises, and heart rate for warming up and walking down were lower than these intensities. Each training session consisted of a five-minute stretching program, 10 to 15-minute dynamic warm-up program, 20 to 30 minutes of core exercises including brisk walking, soft and light running, motion and movement, local endurance and relaxation and so on, finally a ten-minute cooling program and recovery. Maximum heart rate for each subject was calculated using the Formula (220 - age), and participants' heart rate was controlled using a Polar watch (model: Pox 1000, made in Japan). All experiments performed on humans were in accordance with the guidelines of the Ethical Committee for Research on Humans of Urmia University.

\subsection{Data Collection}

The subjects attended the laboratory 48 hours before the first training session and 48 hours after completion of the course, at $8 \mathrm{am}$, after overnight fasting and having been abstained from exercise; $10 \mathrm{~mL}$ fasting venous blood was obtained from the antecubital vein. Blood samples were collected in test tubes and immediately transferred to liquid nitrogen. Participants' weights were measured with a (sensitivity of $0.1 \mathrm{~kg}$ ) digital scale before the initial sampling and at the end of the research program. Heartbeat was constantly monitored by the polar device (F1tm model). Plasma HDL-C was determined by the direct Immunohistochemistry method (HDL-C immuno FS, Pars Azmoon, Karaj). The intra-assay coefficient of variation and sensitivity of the method were $1.2 \%$ and $0.03 \mathrm{mmol} / \mathrm{L}$, respectively. The procedure of Friedewald (17) was used to estimate LDL-C. Fat mass, percentage of body fat and lean mass were assessed with bioelectrical impedance systems (BIA-106, RJL Systems, USA). Total cholesterol, triglycerides and plasma HDL-C was determined by the direct Immunohistochemistry method (HDL-C immuno FS, Pars Azmoon, Karaj). The intra-assay coefficient of variation and sensitivity of the method were $1.2 \%$ and $0.03 \mathrm{mmol} / \mathrm{L}$, respectively. Fasting blood glucose levels were measured by the God Photometric Method (using the glucose kit made by Pars Azmoon with internal measurement degree of 1.28 and sensitivity of $5 \mathrm{mg} / \mathrm{dL}$ ). Total RNA was isolated from peripheral blood using Trizol (Invitrogen, Inc) and 1ug RNA subjected to reverse transcription using the first strand cDNA synthesis kit (Fermentas), according to the manufacturer's instructions. To determine mRNA expression level, the following primers were used for ABCA1, APO-A1 and GAPDH (as an internal control) based on gene specific sequences. ABCA1-Forward: 5'-CAAACATGTCAGCTGCTGCTGGAAG-3', ABCA1-Reverse: 5'-GAGCCTCCCCAGGAGTCG-3'. GAPDH-Forward: 5'TCACCATCTTCCAGGAGCGA-3', GAPDH-Reverse: 5'- AGTGATGGCATGGACTGTGG-3', APO-A1- Forward: 5'-CCTGGGATCGAGTGAAGGAC-3', APO-A1Reverse: 5'-CGTGCTCAGATGCTCGGTGG-3' (18).

Polymerase chain reaction was performed for 28 cycles, denaturationat $94^{\circ} \mathrm{C}$ for 30 seconds, annealing at $55^{\circ} \mathrm{C}$ for 30 seconds and development at $72^{\circ} \mathrm{C}$ for 50 seconds. 
Tofighi A et al.

Table 1. Participants' Characteristics ${ }^{\text {a }}$

\begin{tabular}{|c|c|c|c|c|}
\hline \multirow[t]{2}{*}{ Variable } & \multicolumn{4}{|c|}{ Control Experiment } \\
\hline & T-B & C-A & C-B & T-A \\
\hline BMI, $\mathrm{kg} / \mathrm{m}^{2}$ & $34 \pm 1.48$ & $34 \pm 2.52$ & $34.06 \pm 3.24$ & $31 \pm 3.39^{b}$ \\
\hline Weight, kg & $83.61 \pm 1.49$ & $83.51 \pm 2.21$ & $83.41 \pm 1.28$ & $79.38 \pm 0.14^{b}$ \\
\hline Body fat, \% & $31.4 \pm 3.98$ & $32.56 \pm 4.6$ & $30.59 \pm 3.36$ & $28.49 \pm 2.94^{b}$ \\
\hline $\begin{array}{l}\text { Total cholesterol, mg/ } \\
\text { dL }\end{array}$ & $173.5 \pm 43.9$ & $174.8 \pm 52.9$ & $173.9 \pm 49.3$ & $167.9 \pm 49.7^{b}$ \\
\hline Triglycerides, mg/dL & $171.9 \pm 65.8$ & $171.9 \pm 63.0$ & $172.9 \pm 45.5$ & $155.19 \pm 62.8^{b}$ \\
\hline HDL-C, mg/dL & $43.1 \pm 8.8$ & $44.5 \pm 13.1$ & $43.7 \pm 19.0$ & $46.2 \pm 11.4^{\mathrm{C}}$ \\
\hline LDL-C, mg/dL & $103.9 \pm 29.8$ & $104.9 \pm 43.5$ & $102.1 \pm 10.2$ & $94.7 \pm 52.5^{b}$ \\
\hline Glucose, mmol/L & $8.7 \pm 0.7$ & $9.1 \pm 0.8$ & $8.8 \pm 0.6$ & $5.8 \pm 0.7^{\mathrm{C}}$ \\
\hline
\end{tabular}

a Data are shown as Means \pm SD.

b Significance of change at level of 0.05 .

c Significance of change at level of 0.001 .

Template cDNA was standardized by reinforcement of a 700-bp internal control of GAPDH, a house keeping gene. All the reactions were repeated for a minimum of three times to ensure repeatability. ABCA1 cDNA was amplified giving a 211-bp product, and APO-A1 cDNA was amplified giving a 587-bp product. All PCR products were electrophoresed on agarosegel and bands were visualized by gel documentation (Pro logic 212 imaging system, Carestream, USA). The intensity of the bands was measured by computer-integrated densitometry (Pro logic 212 imaging system, Carestream, USA). Levels of mRNA were expressed as ratios.

\subsection{Statistical Analysis}

All results are expressed as means \pm SD. All variables were compared by unpaired t-test. All statistical analysis was performed using the SPSS software (version 18).

\section{Results}

Analysis of biochemical parameters in aerobic exercise groups are shown in Table 1 . The control group did not show any significant difference after the period of training in any of the estimated parameters. However, Body Mass Index (BMI) levels decreased significantly in the experimental group after training $(31 \pm 3.39)$ compared to before training $(34 \pm 1.48)(\mathrm{P}<0.05)$. Similarly, fat percentage analysis was significantly differentafter training $(28.49 \pm 2.94)$ compared to levels before training (31.4 \pm 3.98 ) in the experimental group. Also, the weight parameter was significantly different after training (79.38 $\pm 0.14)$ compared to before training $(83.61 \pm 1.49)$ in the experimental group. Total cholesterol and triglyceride levels were also significantly lower after training (167.9 \pm 49.7 and $155.19 \pm 62.8$ ) compared to before training (173.5 \pm 43.9 and $171.9 \pm 65.8)$ in the experimental group $(\mathrm{P}<$ $0.05)$. Interestingly, aerobic training caused a significant increase in the HDL levels of the experimental group ( $P$ $<0.05)$ while LDL and glucose levels decreased signifi- cantly after training $(5.8 \pm 0.7$ and $94.7 \pm 52.5)$ compared to before training $(8.7 \pm 0.7$ and $103.9 \pm 29.8)(\mathrm{P}<0.05)$. Data analysis revealed that the expression of ABCA1 in blood remained unchanged in the control group, yet there was a significant difference between post and pre ABCA1 (Figure 1 and Figure $2 \mathrm{~A}$ ) and APO-A1 (Figure 1 and Figure 2 B) gene expression levels in the training group, (ABCA1; $\mathrm{P}=$ $0.036, \mathrm{t}=2.74$ ), (APO-A1; $\mathrm{P}=0.04, \mathrm{t}=3.20$ ) (Figure $2 \mathrm{~A}$ and Figure 2 B).

Figure 1. Identification of Amplified Products by Real Time-Polymerase Chain Reaction With the Method of Agarose Gel Electrophoresis

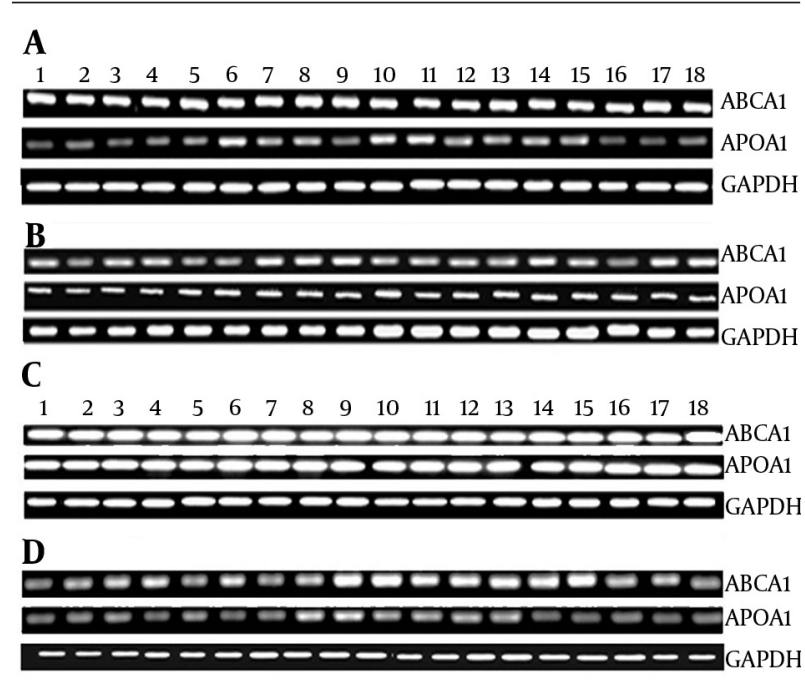

Duplex RT-PCR was performed on RNA with primers generating fragments of 211, 587 and 700 bp for ABCA1, APO-A1 and GAPDH, respectively. The GAPDH was used as an internal control. A, Test before training; B, Control before training; C, Test after training and D, Control after training. Different levels of transcript were seen in control and test individuals. Both ABCA1 and APOA1 were found in control and test groups before and after training with alternative transcripts being highly up regulated in test individuals after 12 weeks of training. 
Figure 2. The Change of $A, A B C A 1$, and $B, A P O A 1$, Transcript Levels in the Blood of 18 Control and Test Individuals Before and After Twelve Weeks of Aerobic Training

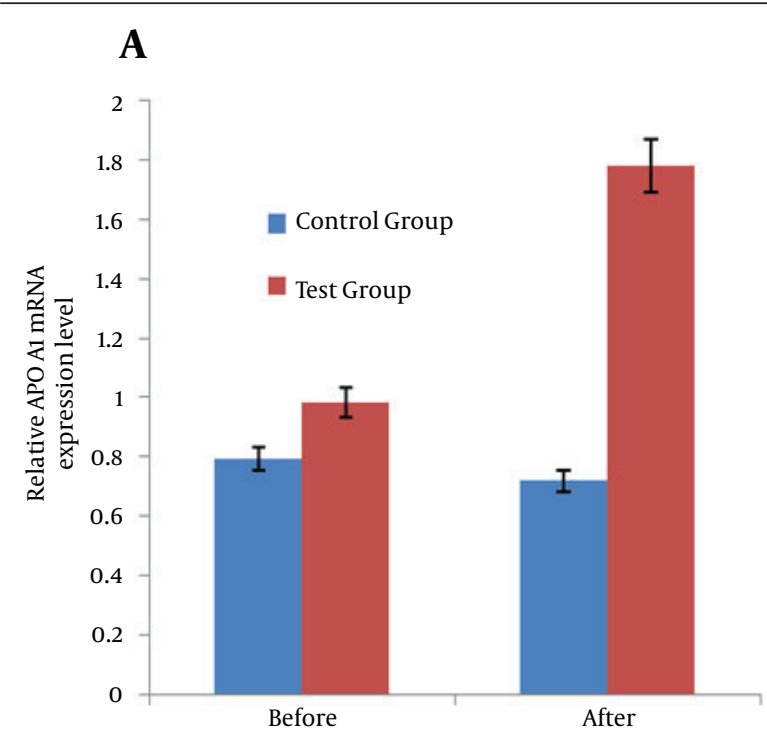

B

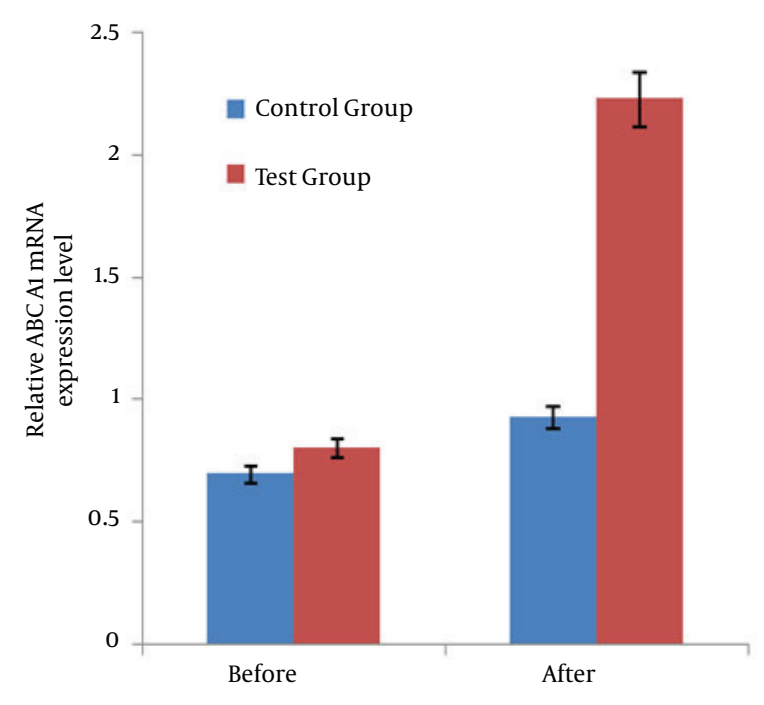

The ABCA1 mRNA level was measured by densitometry and normalized to GAPDH mRNA expression level. Values are representative of ABCA1/GAPDH expression level.

\section{Discussion}

The results of this study show that 12 weeks of aerobic exercise attended by inactive women significantly reduced LDL, glucose, triglycerides and total cholesterol while it increased ABCA1 and Apo-A1 expression. Previous studies have demonstrated beneficial effects of exercise on ABCA1 expression $(13,19,20)$. Moreover, the effect of exercise training programs on RCT and its main factors exclusive of ABCA1 has been reviewed by several researchers, yet to the best of our knowledge this is the fourth human research to show that ABCA1 is expressed due to exercise in peripheral blood $(12,15)$. The results of the current study showed that twelve weeks of regular aerobic exercise with $65-75 \%$ of HRmax, increased ABCA1 expression of blood in trained women. Plasma HDL-C levels increased following twelve weeks of aerobic training. Furthermore, plasma LDL-C levels decreased significantly following the protocol. Our findings on ABCA1 expression are somewhat similar to previous findings reported by Ghanbari-Niaki et al. (15). They investigated the expression of ABCA1 mRNA in peripheral blood and found a significant increase shortly after exercise at all given exercise intensities ( $40 \%, 60 \%$ and $80 \%$ of 1 RM) with more noticeable results for $60 \%$ of the group. GhanbariNiaki et al. reported that ABCA1 was expressed in blood after exercise of different intensities (40\%,60\% and $80 \%$ of $1 \mathrm{RM})(15)$. ABCA1 is a cell membrane transporter that facilitates delivery of phospholipids from cell membranes to lipid poor apoA- 1 with the formation of discoidalapoAI-containing HDL, and it plays a central role in formation of plasma HDL. Hoang et al. reported that participants with high physical activity had higher levels of ABCA1 expression (12). According to Butcher et al. a low-intensity training program (eight weeks of low-intensity exercise program consisting of walking 10000 steps, three times a week) resulted in a 3.46 and 3.06 fold increase in blood ABCA1 and ABCG1 expression (16). Our Data of plasma HDL-C and LDL-C concentrations were also in line with several previous reports (21-23). In the study of Olchawa et al. 25 endurance-trained male athletes were compared with 33 males enjoying an active lifestyle. Plasma concentrations of HDL-C and ApoA-I were higher in athletes compared with active participants (24). A meta-analysis carried out by Kelley et al. on human subjects, revealed that aerobic exercise causes significant reduction of blood glucose concentration, total cholesterol (TC), LDL-C, triglyceride (TG) and BMI whereas an increase in HDL-C level (25). Mukhopadhyay et al. also reported that participants who performed aerobic activities showed a significant decrease of LDL-C and TC, while an increase in HDL-C, VLDL and TG levels (26). Hepatic over expression of ABCA1 in mice led to an increase of plasma HDL-C levels. In contrast, apoA-I and plasma HDL levels are considerably reduced in mice with a liver-specific elimination of ABCA1 $(27,28)$. Therefore, hepatic expression of ABCA1 is a rate-limiting factor for production of plasma HDL (29). Although ABCA1 in macrophages has little influence on plasma HDL levels, it is a major factor in the prevention of excessive cholesterol agglomeration in macrophages of the arterial wall and evolution of foam cells, independent of plasma HDL levels. Our intervention resulted in a significant increase in ABCA1 and APOA1 expression, decrease in TC and TG levels, and an increase in HDL-C and LDL-C. Therefore, it could be suggested that an aerobic program can improve TC, TG, and HDL-C levels. In conclusion, the results of this study suggest that aerobic exercise would be appropriate for increasing HDL-C and reducing TC/ HDL-C with low cost and minimal side effects. 


\subsection{Practical Implications}

- Aerobic exercises can increase the expression of ABCA1and APO-A1 genes, which are effective factors in the prevention of cardiovascular disease.

- Aerobic program participation also improves TC, TG, and HDL-C levels and reduces TC/HDL-C.

- Aerobic exercises can improve health with low cost and minimal side effects.

\section{Acknowledgements}

The authors gratefully thank Mr. Farhang Pazhoh for his technical assistance of this work.

\section{Authors' Contributions}

Study concept and design: Asghar Tofighi, Solmaz Babaei and Fatemeh Rahmani. Analysis and interpretation of data: Asghar Tofighi, Bahram Jamali Qarakhanlou and Solmaz Babaei. Drafting of the manuscript: Asghar Tofighi and Fatemeh Rahmani. Critical revision of the manuscript for important intellectual content: Fatemeh Rahmani, Bahram Jamali Qarakhanlou and Solmaz Babaei. Statistical analysis: Asghar Tofighi, Fatemeh Rahmani and Bahram Jamali Qarakhanlou.

\section{References}

1. Rashidlamir A, Ghanbari-Niaki A, Saadatnia A. The Effect of eight weeks of wrestling and wrestling technique based circuit training on lymphocyte ABCA1 gene expression and plasma apolipoprotein AI. World J Sport Sci. 2011;2(2):144-50.

2. Morgan J, Carey C, Lincoff A, Capuzzi D. High-density lipoprotein subfractions and risk of coronary artery disease. Curr Atheroscler Rep. 2004;6(5):359-65.

3. Yancey PG, Bortnick AE, Kellner-Weibel G, de la Llera-Moya M, Phillips MC, Rothblat GH. Importance of different pathways of cellular cholesterol efflux. Arterioscler Thromb Vasc Biol. 2003;23(5):712-9.

4. Cavelier C, Lorenzi I, Rohrer L, von Eckardstein A. Lipid efflux by the ATP-binding cassette transporters ABCA1 and ABCG1. Biochim Biophys Acta. 2006;1761(7):655-66.

5. Khabazian BM, Ghanbari-Niaki A, Safarzadeh-Golpordesari A, Ebrahimi M, Rahbarizadeh F, Abednazari H. Endurance training enhances ABCA1 expression in rat small intestine. Eur J Appl Physiol. 2009;107(3):351-8.

6. Langmann T, Klucken J, Reil M, Liebisch G, Luciani MF, Chimini $\mathrm{G}$, et al. Molecular cloning of the human ATP-binding cassette transporter 1 (hABC1): evidence for sterol-dependent regulation in macrophages. Biochem Biophys Res Commun. 1999;257(1):29-33.

7. Sahoo D, Trischuk TC, Chan T, Drover VA, Ho S, Chimini G, et al. ABCA1-dependent lipid efflux to apolipoprotein A-I mediates HDL particle formation and decreases VLDL secretion from murine hepatocytes. J Lipid Res. 2004;45(6):1122-31.

8. Timmins JM, Lee JY, Boudyguina E, Kluckman KD, Brunham LR, Mulya A, et al. Targeted inactivation of hepatic Abca1 causes profound hypoalphalipoproteinemia and kidney hypercatabolism of apoA-I. J Clin Invest. 2005;115(5):1333-42.

9. Singaraja RR, Van Eck M, Bissada N, Zimetti F, Collins HL, Hildebrand RB, et al. Both hepatic and extrahepatic ABCA1 have discrete and essential functions in the maintenance of plasma high-density lipoprotein cholesterol levels in vivo. Circulation.
2006;114(12):1301-9.

10. Singaraja RR, James ER, Crim J, Visscher H, Chatterjee A, Hayden MR. Alternate transcripts expressed in response to diet reflect tissue-specific regulation of ABCA1. J Lipid Res. 2005;46(10):206171.

11. Ghanbari-Niaki A, Khabazian BM, Hossaini-Kakhak SA, Rahbarizadeh F, Hedayati M. Treadmill exercise enhances ABCA1 expression in rat liver. Biochem Biophys Res Commun. 2007;361(4):841-6.

12. Hoang A, Tefft C, Duffy SJ, Formosa M, Henstridge DC, Kingwell BA, et al. ABCA1 expression in humans is associated with physical activity and alcohol consumption. Atherosclerosis. 2008;197(1):197-203.

13. Zieker D, Fehrenbach E, Dietzsch J, Fliegner J, Waidmann M, Nieselt $\mathrm{K}$, et al. cDNA microarray analysis reveals novel candidate genes expressed in human peripheral blood following exhaustive exercise. Physiol Genomics. 2005;23(3):287-94.

14. Oram JF, Vaughan AM. ATP-Binding cassette cholesterol transporters and cardiovascular disease. Circ Res. 2006;99(10):1031-43.

15. Ghanbari-Niaki A, Saghebjoo M, Hedayati M. A single session of circuit-resistance exercise effects on human peripheral blood lymphocyte ABCA1 expression and plasma HDL-C level. Regul Pept. 2011;166(1-3):42-7.

16. Butcher LR, Thomas A, Backx K, Roberts A, Webb R, Morris K. Lowintensity exercise exerts beneficial effects on plasma lipids via PPARgamma. Med Sci Sports Exerc. 2008;40(7):1263-70.

17. Orlov SV, Mogilenko DA, Shavva VS, Dizhe EB, Ignatovich IA, Perevozchikov AP. Effect of TNFalpha on activities of different promoters of human apolipoprotein A-I gene. Biochem Biophys Res Commun. 2010;398(2):224-30.

18. Connolly PH, Caiozzo VJ, Zaldivar F, Nemet D, Larson J, Hung SP, et al. Effects of exercise on gene expression in human peripheral blood mononuclear cells. J Appl Physiol (1985). 2004;97(4):1461-9.

19. Buttner P, Mosig S, Lechtermann A, Funke H, Mooren FC. Exercise affects the gene expression profiles of human white blood cells.J Appl Physiol (1985). 2007;102(1):26-36.

20. Gordon PM, Fowler S, Warty V, Danduran M, Visich P, Keteyian S. Effects of acute exercise on high density lipoprotein cholesterol and high density lipoprotein subfractions in moderately trained females. BrJ Sports Med.1998;32(1):63-7.

21. Sgouraki E, Tsopanakis A, Tsopanakis C. Acute exercise: response of HDL-C, LDL-C lipoproteins and HDL-C subfractions levels in selected sport disciplines. J Sports Med Phys Fitness. 2001;41(3):386-91.

22. Visich PS, Goss FL, Gordon PM, Robertson RJ, Warty V, Denys BG, et al. Effects of exercise with varying energy expenditure on highdensity lipoprotein-cholesterol. Eur J Appl Physiol Occup Physiol. 1996;72(3):242-8.

23. Angelopoulos TJ, Robertson RJ, Goss FL, Metz KF, LaPorte RE. Effect of repeated exercise bouts on high density lipoproteincholesterol and its subfractions HDL2-C and HDL3-C. Int J Sports Med.1993;14(4):196-201.

24. Olchawa B, Kingwell BA, Hoang A, Schneider L, Miyazaki O, Nestel $\mathrm{P}$, et al. Physical fitness and reverse cholesterol transport. Arterioscler Thromb Vasc Biol. 2004;24(6):1087-91.

25. Kelley GA, Kelley KS, Tran ZV. Exercise, lipids, and lipoproteins in older adults: a meta-analysis. Prev Cardiol. 2005;8(4):206-14.

26. Mukhopadhyay SK. Study of lipid profile in obese individuals and the effect of cholesterol lowering agents on them. A meen J Med Sci . 2012;5(2):147-51.

27. Singaraja RR, Fievet C, Castro G, James ER, Hennuyer N, Clee SM, et al. Increased ABCA1 activity protects against atherosclerosis. $J$ Clin Invest. 2002;110(1):35-42.

28. Haghpassand M, Bourassa PA, Francone OL, Aiello RJ. Monocyte/ macrophage expression of ABCA1 has minimal contribution to plasma HDL levels. J Clin Invest. 2001;108(9):1315-20.

29. Aiello RJ, Brees D, Bourassa PA, Royer L, Lindsey S, Coskran T, et al. Increased atherosclerosis in hyperlipidemic mice with inactivation of ABCA1 in macrophages. Arterioscler Thromb Vasc Biol. 2002;22(4):630-7. 